\title{
Evaluation of the in vitro activity of ceftobiprole against clinical isolates of Staphylococcus aureus
}

\author{
Carola Mauri, Silvia Bracco, Elisa Meroni, Davide Oggioni, Luigi Principe, Beatrice Pini, \\ Francesco Luzzaro
}

Microbiology and Virology Unit, A. Manzoni Hospital, Lecco, Italy

\section{Summary}

Background and aims: Ceftobiprole is a new cephalosporin characterized by a potent activity against Gram-positive and Gram-negative bacterial pathogens. It is noting that ceftobiprole has a strong affinity for penicillin binding proteins including PBP 2A, which mediates resistance to beta-lactams in methicillin (oxacillin)-resistant coagulase-negative staphylococci and Staphylococcus aureus (MRSA). The aim of the current study was to examine the antimicrobial activity of ceftobiprole against clinical isolates of $S$. aureus recently collected at our institution.

Materials and methods: One hundred and forty blood isolates of $S$. aureus were evaluated, including methicillin-susceptible (MSSA, $\mathrm{n}=70$ ) and MRSA ( $\mathrm{n}=70)$ strains. Twenty additional MRSA isolates obtained from different sites (including skin and soft tissues, blood,

Correspondence: Francesco Luzzaro, Laboratorio di Microbiologia e Virologia, Ospedale Alessandro Manzoni, Via dell'Eremo 9/11, 23900 Lecco, Italy.

Tel.: +39.0341 .489630 - Fax: +39.0341 .489601 .

E-mail: f.luzzaro@asst-lecco.it

Key words: MRSA; ceftobiprole; new antibiotic; new cephalosporin, S. aureus treatment; anti-MRSA activity.

Contributions: CM, study design, laboratory work, collection of data, analysis and interpretation of data, manuscript preparation; SB, EM and D0, study design, laboratory work, collection of data, analysis and interpretation of data; LP and BP, study design, analysis and interpretation of data, critical revision of the manuscript; FL, study design, analysis and interpretation of data, critical revision of the manuscript, final approval of the version to be submitted for publication.

Conflict of interest: the authors declare no potential conflict of interest.

Received for publication: 27 July 2016.

Revision received: 14 September 2016.

Accepted for publication: 18 September 2016.

(C) Copyright C. Mauri et al., 2016

Licensee PAGEPress, Italy

Microbiologia Medica 2016; 31:6205

doi:10.4081/mm.2016.6205

This article is distributed under the terms of the Creative Commons Attribution Noncommercial License (by-nc 4.0) which permits any noncommercial use, distribution, and reproduction in any medium, provided the original author(s) and source are credited. and lower respiratory tract) and characterized by borderline susceptibility to vancomycin were also studied to assess the ability of ceftobiprole to overcome this worrisome trait. MIC values of ceftobiprole were determined by Etest strips and results were interpreted according to EUCAST guidelines.

Results and conclusions: Study isolates were consistently susceptible to ceftobiprole, with MIC values ranging from $0.125 \mathrm{mg} / \mathrm{L}$ to $2 \mathrm{mg} / \mathrm{L}$. Overall, $\mathrm{MIC}_{50}$ and $\mathrm{MIC}_{90}$ were $0.25 \mathrm{mg} / \mathrm{L}$ and $0.5 \mathrm{mg} / \mathrm{L}$, respectively. Ceftobiprole showed in vitro activity against all $S$. aureus isolates, with small differences among groups selected on the basis of resistance to methicillin and/or reduced susceptibility to vancomycin. Thus, ceftobiprole appears a valid choice for treating infections caused by $S$. aureus, even when susceptibility results are not yet available. Additionally, ceftobiprole may be a valid option in the case of reduced susceptibility to vancomycin.

\section{Introduction}

Staphylococcus aureus is a Gram-positive bacterium that can live as a commensal organism on the skin, in the nose and throat. Approximately $30 \%$ of healthy people are asymptomatically colonized by $S$. aureus, which permanently colonizes the anterior nares in 10$20 \%$ of the population and intermittently colonizes $30-50 \%$; the rest of the population never becomes colonized $(3,21)$. Importantly, this colonization is a known risk factor for infection $(10,19,28,31,35)$. Transmission results most frequently from transient colonization through the hands of hospital staff that carry strains from one patient to another $(6,23)$. The vast majority of people who develop infections caused by $S$. aureus are infected with their own colonizing strains; however, these infections can also be obtained from other people or environmental exposures. $S$. aureus is a prominent human pathogen that can cause a diverse range of diseases ranging from relatively minor skin infections to serious and life-threatening infections such as endocarditis, pneumonia, and sepsis (21).

Resistance to antibiotics is a well-known serious problem in medicine. $S$. aureus has a great ability to acquire resistance to antibiotics. In 1961, Patricia Jevons reported the first isolates of methicillin-resis$\operatorname{tant} S$. aureus (MRSA), only 2 years after the clinical usage of methicillin $(24,33)$. In the following decades, MRSA isolates have spread throughout the world and can be found nowadays in many industrialized countries (6).

Resistance to methicillin is primarily the result of decreased binding affinity to its target, penicillin binding protein 2 (PBP2), owing to acquisition of an altered PBP2 (PBP2a) encoded by the $m e c A$ gene harboured on a mobile genetic element, the staphylococcal chromosome cassette mec (SCCmec) $(16,22)$. PBP2a has a low $\beta$-lactam affinity and confers resistance to most molecules of this family (including 
third- and fourth-generation cephalosporins, and carbapenems). Because of its low $\beta$-lactam affinity, PBP2a can mediate cell wall assembly when the normal staphylococcal PBPs are blocked by these compounds (11). In addition to most $\beta$-lactam antibiotics, MRSA are usually resistant to many other antibiotics, such as erythromycin, gentamycin, ciprofloxacin, and fusidic acid (27). So, methicillin resistance is an indicator of resistance to a wide range of antibiotics. The continued increase of MRSA has led to a concurrent increase in reliance on non- $\beta$-lactam agents for treatment, especially vancomycin (14). Borderline susceptibility to this drug (i.e., isolates having MIC values for vancomycin ranging from 1.5 to $2 \mathrm{mg} / \mathrm{L}$ ) have been increasingly reported, often associated to therapeutic failures $(15,32)$. Alternative agents that have regulatory approval for some MRSA infections include linezolid, tigecycline, and daptomycin (20). All of them have been approved for skin and soft tissue infections (SSTIs). In addition, due to their pharmacokinetic and pharmacodynamics characteristics, linezolid is also indicated especially for pneumonia, whereas daptomycin is often used in the treatment of bloodstream infections, including rightsided infective endocarditis $(29,30,34)$. These molecules can be used for specific infections, taking into account their toxicity and the emerging increase of resistance determinants (26).

Ceftobiprole is a new broad-spectrum $\beta$-lactam antimicrobial agent belonging to cephalosporins (Figure 1), intravenously administered as a prodrug (ceftobiprole medocaril) and characterized by a potent activity against both Gram-positive and Gram-negative bacterial pathogens. Of note, it has anti-MRSA activity. Similarly to other $\beta$-lactam antibacterial agents, ceftobiprole exerts its antibacterial activity by binding to important penicillin-binding proteins (PBPs) and inhibiting their transpeptidase activity (12), which is essential for the synthesis of the peptidoglycan layer of bacterial cell walls. Ceftobiprole binds to multiple PBPs in clinically relevant pathogens, which provides its broad activity spectrum $(4,5,7,12,13)$. The anti-MRSA activity of ceftobiprole is attributed to its rapid and tight binding to the mutant PBP2a form (encoded by the mecA gene) that is able to confer resistance to methicillin and most $\beta$-lactam antibiotics (12). Ceftobiprole is the first cephalosporin monotherapy approved in the EU for the treatment of both hospital(HAP) and community-acquired pneumonia (CAP), excluding ventilator-associated pneumonia (VAP).

The aim of this study was to evaluate the activity of ceftobiprole against invasive isolates (obtained from blood cultures) of both methicillin-susceptible $S$. aureus (MSSA) and MRSA strains. In addition, MRSA isolates recovered from different sites and characterized by borderline susceptibility to vancomycin were evaluated.

\section{Materials and Methods}

A total of 160 non duplicate, clinical isolates of $S$. aureus previously collected at our Institution were evaluated against ceftobiprole and other antimicrobial agents. One hundred and forty blood isolates of $S$. aureus were evaluated, including MSSA $(\mathrm{n}=70)$ strains. Twenty additional MRSA isolates obtained from different sites and characterized by borderline susceptibility to vancomycin (MICs, $1.5-2 \mathrm{mg} / \mathrm{L}$ ) were also studied to verify the ability of ceftobiprole to overcome this worrisome trait. These isolates were recovered from skin and soft tissues $(n=7)$, surgical wounds $(n=6)$, lower respiratory tract secretions $(n=5)$, blood $(n=1)$ and prosthetic joint infection $(n=1)$. Bacterial identification and antimicrobial susceptibility were routinely obtained by MALDI-TOF mass spectrometry (Vitek® MS, bioMérieux, Marcy l'Étoile, France) and Vitek® 2 instrument (bioMérieux), respectively. Isolates characterized by borderline susceptibility to vancomycin were further evaluated by Etest ${ }^{\circledR}$ strips (bioMérieux) to confirm vancomycin MIC values. $S$. aureus ATCC 29213 was used as control. Susceptibility results were interpreted on the basis of EUCAST breakpoints (8). Starting from frozen isolates, collection strains were first inoculated on Columbia blood agar (bioMérieux) to resume their metabolic activity. After $18 \mathrm{~h}$ incubation at $36^{\circ} \mathrm{C}$, a $0.5 \mathrm{McFarland}$ suspension for each isolate was prepared in sterile $0.9 \%$ saline (corresponding to a cell density of $1.5 \times 10^{8} \mathrm{CFU} / \mathrm{mL}$ ) and inoculated on Mueller Hinton $\mathrm{E}$ agar (bioMérieux). MIC values of ceftobiprole were determined by Etest (MIC range, 0.002-32 mg/L), a well-established method for antimicrobial susceptibility testing in microbiology laboratories. According to EUCAST breakpoints, isolates of $S$. aureus having MICs $\leq 2 \mathrm{mg} / \mathrm{L}$ were interpreted as susceptible to ceftobiprole (8).

\section{Results}

Study isolates were consistently susceptible to ceftobiprole, with MIC values ranging from $0.125 \mathrm{mg} / \mathrm{L}$ to $2 \mathrm{mg} / \mathrm{L}$. Overall, the ceftobiprole $\mathrm{MIC}_{50}$ and $\mathrm{MIC}_{90}$ values (MIC required to inhibit $50 \%$ and $90 \%$ of the isolates) were $0.25 \mathrm{mg} / \mathrm{L}$ and $0.5 \mathrm{mg} / \mathrm{L}$, respectively, with small variations within different groups (Table 1). The overall modal MIC value was $0.5 \mathrm{mg} / \mathrm{L}$ (45 isolates; 28.1\%). The MIC distribution of MSSA and MRSA groups are shown in Figure 2. In particular, $\mathrm{MIC}_{50}$ and $\mathrm{MIC}_{90}$ for the different groups were as follows: MSSA, $0.25 \mathrm{mg} / \mathrm{L}$ and $0.5 \mathrm{mg} / \mathrm{L} ;$ MRSA, $1 \mathrm{mg} / \mathrm{L}$ and $1.5 \mathrm{mg} / \mathrm{L}$. For the different groups the modal MIC was $0.5 \mathrm{mg} / \mathrm{L}$ (27 isolates; $38.6 \%)$ and $1 \mathrm{mg} / \mathrm{L}$ (32 isolates; 35.6\%) for MSSA and MRSA, respectively. MSSA isolates showed low MIC values for ceftobiprole, ranging from 0.125 to $0.5 \mathrm{mg} / \mathrm{L}$. These strains were also susceptible to most of tested antibiotics, with very low resistance rates for erythromycin (11.4\%), levofloxacin (7.1\%) and gentamicin (5.7\%). Concerning MRSA, these strains were characterized by MIC values for ceftobiprole higher than those observed for MSSA, with a range of 0.125-2 mg/L. High resistance rates among MRSA were observed for levofloxacin (94.3\%) and erythromycin (57.1\%), while gentamicin and tetracycline resistance was observed in few cases (8.6\%).
Prodrug: ceftobiprole medocaril

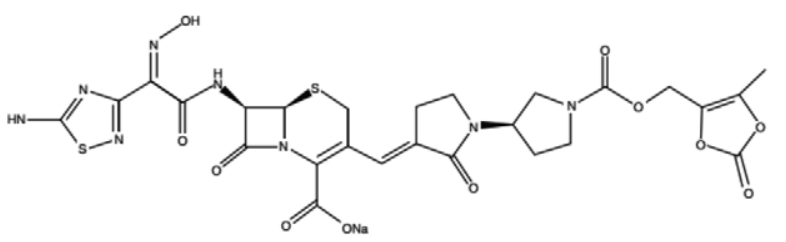

Active metabolite: ceftobiprole

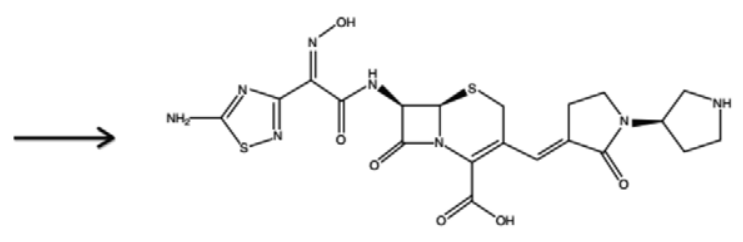

Figure 1. Chemical structure of the prodrug ceftobiprole medocaril and its active metabolite, ceftobiprole. 
Regarding the vancomycin-borderline MRSA subgroup, these isolates were characterized by $\mathrm{MIC}_{50}$ and $\mathrm{MIC}_{90}$ values for ceftobiprole $(0.5$ $\mathrm{mg} / \mathrm{L}$ and $1 \mathrm{mg} / \mathrm{L}$, respectively) intermediate between those observed for MSSA and MRSA, with a range of 0.125-2 mg/L. High resistance rates among vancomycin-borderline MRSA were observed not only for levofloxacin (85.0\%) and erythromycin (80.0\%) but also for gentamicin (60.0\%), teicoplanin (50.0\%) and tetracycline (35.0\%). In vitro activity of ceftobiprole and comparator agents against study isolates is summarized in Table 1. Of note, vancomycin, linezolid, and tigecyline showed $100 \%$ susceptibility independently on different groups.

\section{Discussion and Conclusions}

Ceftobiprole is a fifth-generation cephalosporin, newly approved in Europe as a single-agent therapy for HAP and CAP. Of note, it is an extended-spectrum cephalosporin that has been reported to encompass activity against both Gram-negative and Gram-positive strains, including MRSA. In our experience, focused on $S$. aureus clinical isolates, ceftobiprole showed in vitro activity against all $S$. aureus isolates (MIC values $\leq 2 \mathrm{mg} / \mathrm{L} ; 100 \%$ susceptible), with small differences among

Table 1. Activity of ceftobiprole and comparator agents tested against 160 Staphylococcus aureus clinical isolates.

\begin{tabular}{|c|c|c|c|c|}
\hline \multirow[t]{2}{*}{ S. aureus groups (no. tested) } & \multicolumn{2}{|c|}{ MIC (mg/L) } & \multirow[t]{2}{*}{ MIC range } & \multirow[t]{2}{*}{$\% \mathrm{~S}$} \\
\hline & $\mathrm{MIC}_{50}$ & $\mathrm{MIC}_{90}$ & & \\
\hline \multicolumn{5}{|l|}{ All $S$. aureus (160) } \\
\hline Ceftobiprole & 0.25 & 0.5 & $0.125-2$ & 100 \\
\hline Vancomycin & 1 & 2 & $\leq 0.5-2$ & 100 \\
\hline Linezolid & 2 & 4 & $1-4$ & 100 \\
\hline Tigecycline & $\leq 0.12$ & 0.25 & $\leq 0.12-0.5$ & 100 \\
\hline Co-trimoxazole & $\leq 10$ & $\leq 10$ & $\leq 10$ to $\geq 320$ & 98.8 \\
\hline Teicoplanin & $\leq 0.5$ & 4 & $\leq 0.5-8$ & 93.7 \\
\hline Rifampicin & $\leq 0.03$ & $\geq 4$ & $\leq 0.03$ to $\geq 4$ & 93.1 \\
\hline Tetracycline & $\leq 1$ & $\geq 16$ & $\leq 1$ to $\geq 16$ & 90.6 \\
\hline Gentamicin & $\leq 0.5$ & $\geq 16$ & $\leq 0.5$ to $\geq 16$ & 86.2 \\
\hline Erythromycin & 1 & $\geq 8$ & $\leq 0.25$ to $\geq 8$ & 60.0 \\
\hline Levofloxacin & 4 & $\geq 8$ & $\leq 0.12$ to $\geq 8$ & 45.0 \\
\hline \multicolumn{5}{|l|}{ MSSA (70) } \\
\hline Ceftobiprole & 0.25 & 0.5 & $0.125-0.5$ & 100 \\
\hline Vancomycin & 1 & 1 & $\leq 0.5-1$ & 100 \\
\hline Linezolid & 2 & 2 & $1-2$ & 100 \\
\hline Tigecycline & $\leq 0.12$ & $\leq 0.12$ & $\leq 0.12-0.25$ & 100 \\
\hline Co-trimoxazole & $\leq 10$ & $\leq 10$ & $\leq 10$ to $\geq 320$ & 97.1 \\
\hline Teicoplanin & $\leq 0.5$ & $\leq 0.5$ & $\leq 0.5$ & 100 \\
\hline Rifampicin & $\leq 0.03$ & $\leq 0.03$ & $\leq 0.03$ & 100 \\
\hline Tetracycline & $\leq 1$ & $\leq 1$ & $\leq 1$ to $\geq 16$ & 97.1 \\
\hline Gentamicin & $\leq 0.5$ & $\leq 0.5$ & $\leq 0.5$ to $\geq 16$ & 94.3 \\
\hline Erythromycin & 1 & $\geq 8$ & $\leq 0.25$ to $\geq 8$ & 88.6 \\
\hline Levofloxacin & $\leq 0.12$ & 0.25 & $\leq 0.12$ to $\geq 8$ & 92.9 \\
\hline \multicolumn{5}{|l|}{ MRSA (70) } \\
\hline Ceftobiprole & 1 & 1.5 & $0.125-2$ & 100 \\
\hline Vancomycin & $\leq 0.5$ & 1 & $\leq 0.5-1$ & 100 \\
\hline Linezolid & 2 & 2 & 1-4 & 100 \\
\hline Tigecycline & $\leq 0.12$ & 0.25 & $\leq 0.12-0.25$ & 100 \\
\hline Co-trimoxazole & $\leq 10$ & $\leq 10$ & $\leq 10$ & 100 \\
\hline Teicoplanin & $\leq 0.5$ & $\leq 0.5$ & $\leq 0.5$ & 100 \\
\hline Rifampicin & $\leq 0.03$ & $\leq 0.03$ & $\leq 0.03$ to $\geq 4$ & 97.1 \\
\hline Tetracycline & $\leq 1$ & $\leq 1$ & $\leq 1$ to $\geq 16$ & 91.4 \\
\hline Gentamicin & $\leq 0.5$ & $\leq 0.5$ & $\leq 0.5$ to $\geq 16$ & 91.4 \\
\hline Erythromycin & $\geq 8$ & $\geq 8$ & $\leq 0.25$ to $\geq 8$ & 42.9 \\
\hline Levofloxacin & $\geq 8$ & $\geq 8$ & $\leq 0.12$ to $\geq 8$ & 5.7 \\
\hline \multicolumn{5}{|l|}{ Vancomycin-borderline MRSA (20) } \\
\hline Ceftobiprole & 0.5 & 1 & $0.125-2$ & 100 \\
\hline Vancomycin & 2 & 2 & 2 & 100 \\
\hline Linezolid & 2 & 2 & $2-4$ & 100 \\
\hline Tigecycline & $\leq 0.12$ & $\leq 0.12$ & $\leq 0.12-0.5$ & 100 \\
\hline Co-trimoxazole & $\leq 10$ & $\leq 10$ & $\leq 10$ & 100 \\
\hline Teicoplanin & 2 & 8 & $\leq 0.5-8$ & 50.0 \\
\hline Rifampicin & $\leq 0.03$ & $\geq 4$ & $\leq 0.03$ to $\geq 4$ & 100 \\
\hline Tetracycline & $\leq 1$ & $\geq 16$ & $\leq 1$ to $\geq 16$ & 65.0 \\
\hline Gentamicin & $\geq 16$ & $\geq 16$ & $\leq 0.5$ to $\geq 16$ & 40.0 \\
\hline Erythromycin & $\geq 8$ & $\geq 8$ & $\leq 0.25$ to $\geq 8$ & 20.0 \\
\hline Levofloxacin & $\geq 8$ & $\geq 8$ & 0.25 to $\geq 8$ & 15.0 \\
\hline
\end{tabular}


groups as selected on the basis of methicillin resistance. $\mathrm{MIC}_{50}$ and $\mathrm{MIC}_{90}$ were higher for MRSA than those observed for MSSA (MSSA, 0.25 $\mathrm{mg} / \mathrm{L}$ and $0.5 \mathrm{mg} / \mathrm{L}$; MRSA, $1 \mathrm{mg} / \mathrm{L}$ and $1.5 \mathrm{mg} / \mathrm{L})$. Overall, our data were similar to those found in the literature $(1,2,12,18)$. Specifically, cumulative data from these studies showed that MICs of ceftobiprole for MSSA and MRSA strains ranged from 0.25 to $2 \mathrm{mg} / \mathrm{L}$, and from 0.06 $\mathrm{mg} / \mathrm{L}$ to $2 \mathrm{mg} / \mathrm{L}$, respectively. The $\mathrm{MIC}_{90}$ value was $0.5 \mathrm{mg} / \mathrm{L}$ for MSSA, and $2 \mathrm{mg} / \mathrm{L}$ for MRSA (17).

As also demonstrated by our data, MSSA strains maintain in vitro susceptibility to several antibiotics, whereas a narrow therapeutic arsenal is available for infections caused by MRSA strains. MRSA are frequently difficult to treat because, in addition to $\beta$-lactam antibiotics, they may be resistant to many other commonly used antibiotics, such as erythromycin, gentamicin and levofloxacin (27). Furthermore, the continued increase of MRSA has led to a concurrent increase in reliance on non- $\beta$-lactam agents for treatment, especially vancomycin, a glycopeptides antibiotic with a large number of labeled indications for use $(14,34)$. The main toxicities of vancomycin for concern in critically ill patients include hypersensitivity reactions and renal toxicity. Although vancomycin-resistant $S$. aureus (VRSA) strains are rare, isolates with increased MIC for vancomycin (MIC value ranging from 1.5 to $2 \mathrm{mg} / \mathrm{L}$ ) are common in the clinical practice (15). Importantly, isolates with borderline vancomycin susceptibility are more frequently associated with treatment failures $(15,32)$. Alternative agents that have regulatory approval for some MRSA infections include linezolid, tigecycline, and daptomycin (20).

These drugs, however, present some limitations: can be used for specific infections, are characterized by toxicity and unfortunately, decreased susceptibility and resistance to all of them has been reported $(25,26)$. Taking into account the above limitations of alternative agents for MRSA, ceftobiprole can play an important role for related infections since it is also effective against strains with reduced susceptibility to vancomycin $(1,2,12,18)$. In our experience, MIC distribution of ceftobiprole against vancomycin-borderline MRSA was similar to that of vancomycin-susceptible MRSA isolates, suggesting that decreased susceptibility to vancomycin has a negligible effect on ceftobiprole activity. Similarly, ceftobiprole has been demonstrated to maintain activity against resistant subset of MRSA, including those that were not susceptible to daptomycin, linezolid, or vancomycin (9).

In conclusion, the availability of antibiotics with anti-MRSA activity

\section{口MSSA $\square$ MRSA*}

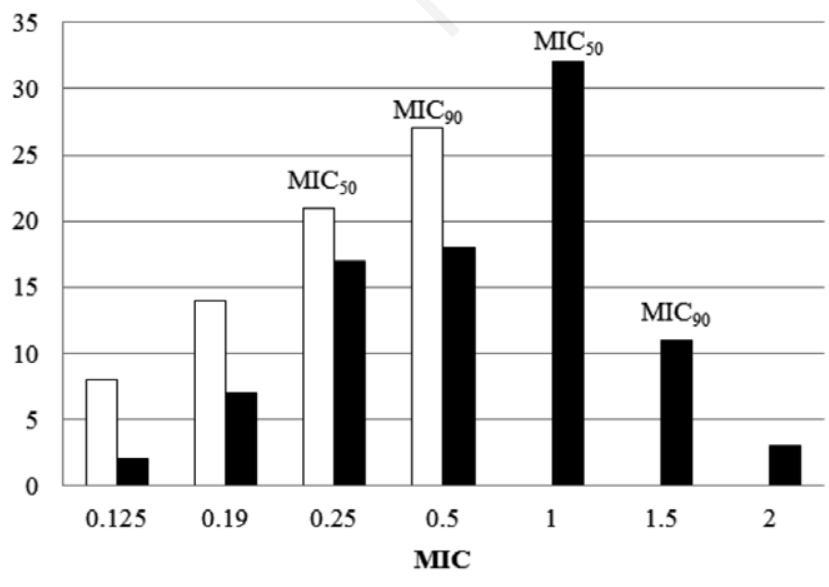

Figure 2. MIC distribution of MSSA and MRSA for ceftobiprole. Tags indicate MIC $_{50}$ and MIC $_{90}$ values. ${ }^{*}$ MRSA group includes isolates characterized by borderline susceptibility to vancomycin. is critical because the prevalence of MRSA infection is increasing worldwide. Ceftobiprole is a new cephalosporin with in vitro broadspectrum activity against most clinically relevant bacterial pathogens, including MRSA thanks to its strong binding to PBP2a. It appears a valid choice for treating infections caused by isolates identified as $S$. aureus even when susceptibility results are not yet available. Additionally, ceftobiprole may be a valid option in the case of reduced susceptibility to vancomycin. The use of ceftobiprole in the clinical practice, increases the therapeutic arsenal against $S$. aureus isolates and helps to reduce the selective pressure that leads to the development of resistance to traditional anti-MRSA antibiotics.

\section{References}

1. Bogdanovich T, Ednie LM, Shapiro S, et al. Antistaphylococcal activity of ceftobiprole, a new broadspectrum cephalosporin. Antimicrob Agents Chemother 2005;49:4210-9.

2. Bozdogan B, Esel D, Whitener C, et al. Antibacterial susceptibility of a vancomycin-resistant Staphylococcus aureus strain isolated at the Hershey Medical Center. J Antimicrob Chemother 2003;52:864-8.

3. Chambers HF. The changing epidemiology of Staphylococcus aureus? Emerg Infect Dis 2001;7:178-82.

4. Davies TA, He W, Bush K, et al. Affinity of ceftobiprole for penicillin-binding protein $2 \mathrm{~b}$ in Streptococcus pneumoniae strains with various susceptibilities to penicillin. Antimicrob Agents Chemother 2010;54:4510-2.

5. Davies TA, Page MG, Shang W, et al. Binding of ceftobiprole and comparators to the penicillin-binding proteins of Escherichia coli, Pseudomonas aeruginosa, Staphylococcus aureus, and Streptococcus pneumoniae. Antimicrob Agents Chemother 2007;51:2621-4.

6. DeLeo FR, Otto M, Kreiswirth BN,et al. Community-associated meticillin-resistant Staphylococcus aureus. Lancet 2010;375:1557-68.

7. Entenza JM, Hohl P, Heinze-Krauss I, et al. BAL9141, a novel extended-spectrum cephalosporin active against methicillin-resistant Staphylococcus aureus in treatment of experimental endocarditis. Antimicrob Agents Chemother 2002;46:171-7.

8. EUCAST. EUCAST Clinical Breakpoints v.5. 2015. European Committee on Antimicrobial Susceptibility Testing. Available frm: http://www.eucast.org/ast_of_bacteria/previous_versions_of_ documents/.

9. Farrell DJ, Flamm RK, Sader HS, et al. Activity of ceftobiprole against methicillin-resistant Staphylococcus aureus strains with reduced susceptibility to daptomycin, linezolid or vancomycin, and strains with defined SCCmec types. Int J Antimicrob Agents 2014;43:323-7.

10. Graham PL 3rd, Lin SX, Larson EL. A US population-based survey of Staphylococcus aureus colonization. Ann Intern Med 2006;144: 318-25.

11. Guignard B, Entenza JM, Moreillon P. Beta-lactams against methicillin-resistant Staphylococcus aureus. Curr Opin Pharmacol 2005;5:479-89.

12 Hebeisen P, Heinze-Krauss I, Angehrn P, et al. In vitro and in vivo properties of Ro 63-9141, a novel broad-spectrum cephalosporin with activity against methicillin-resistant staphylococci. Antimicrob Agents Chemother 2001;45:825-36.

13. Henry X, Verlaine 0 , Amoroso A, et al. Activity of ceftaroline against Enterococcus faecium PBP5. Antimicrob Agents Chemother 2013;57:6358-60.

14. Hersh AL, Chambers HF, Maselli JH, et al. National trends in ambulatory visits and antibiotic prescribing for skin and soft-tissue infections. Arch Intern Med 2008;168:1585-91.

15. Howden BP, Davies JK, Johnson PD, et al. Reduced vancomycin 
susceptibility in Staphylococcus aureus, including vancomycinintermediate and heterogeneous vancomycin-intermediate strains: resistance mechanisms, laboratory detection, and clinical implications. Clin Microbiol Rev 2010;23:99-139.

16. Ito T, Hiramatsu K, Tomasz A, et al. Guidelines for reporting novel mecA gene homologues. Antimicrob Agents Chemother 2012;56:4997-9.

17. Jones ME. In-vitro profile of a new beta-lactam, ceftobiprole, with activity against methicillin-resistant Staphylococcus aureus. Clin Microbiol Infect 2007:17-24.

18. Jones RN, Deshpande LM, Mutnick AH, et al. In vitro evaluation of BAL9141, a novel parenteral cephalosporin active against oxacillinresistant staphylococci. J Antimicrob Chemother 2002;50:915-32.

19. Kuehnert MJ, Kruszon-Moran D, Hill HA, et al. Prevalence of Staphylococcus aureus nasal colonization in the United States, 2001-2002. J Infect Dis 2006;193:172-9.

20. Liu C, Bayer A, Cosgrove SE, et al. Clinical practice guidelines by the infectious diseases society of america for the treatment of methicillin-resistant Staphylococcus aureus infections in adults and children. Clin Infect Dis 2011;52:e18-55.

21. Lowy FD. Staphylococcus aureus infections. N Engl J Med 1998;339:520-32.

22. Matsuhashi M, Song MD, Ishino F, et al. Molecular cloning of the gene of a penicillin-binding protein supposed to cause high resistance to beta-lactam antibiotics in Staphylococcus aureus. J Bacteriol 1986;167:975-80.

23. McCormick JK, Yarwood JM, Schlievert PM. Toxic shock syndrome and bacterial superantigens: an update. Annu Rev Microbiol 2001;55:77-104.

24. Moellering RC Jr. MRSA: the first half century. J Antimicrob Chemother 2012;67:4-11.

25. Munita JM, Bayer AS, Arias CA. Evolving resistance among Grampositive pathogens. Clin Infect Dis 2015:S48-57.
26. Nannini E, Murray BE, Arias CA. Resistance or decreased susceptibility to glycopeptides, daptomycin, and linezolid in methicillinresistant Staphylococcus aureus. Curr Opin Pharmacol 2010;10: 516-21.

27. Otter JA, French GL. Community-associated meticillin-resistant Staphylococcus aureus strains as a cause of healthcare-associated infection. J Hosp Infect 2011;79:189-93.

28. Perl TM, Cullen JJ, Wenzel RP, et al. Intranasal mupirocin to prevent postoperative Staphylococcus aureus infections. N Engl J Med 2002;346:1871-7.

29. Puzniak LA, Quintana A, Wible M, et al. Methicillin-resistant Staphylococcus aureus infection epidemiology and clinical response from tigecycline soft tissue infection trials. Diagn Microbiol Infect Dis 2014;79:261-5.

30. Rybak JM, Barber KE, Rybak MJ. Current and prospective treatments for multidrug-resistant gram-positive infections. Expert Opin Pharmacother 2013;14:1919-32.

31. Shopsin B, Mathema B, Martinez J, et al. Prevalence of methicillinresistant and methicillin-susceptible Staphylococcus aureus in the community. J Infect Dis 2000;182:359-62.

32. Soriano A, Marco F, Martínez JA, et al. Influence of vancomycin minimum inhibitory concentration on the treatment of methicillinresistant Staphylococcus aureus bacteremia. Clin Infect Dis 2008;46:193-200.

33. Sung JY, Lee J, Choi EH,et al. Changes in molecular epidemiology of community-associated and health care-associated methicillinresistant Staphylococcus aureus in Korean children. Diagn Microbiol Infect Dis 2012;74:28-33.

34. Vazquez-Guillamet C, Kollef MH. Treatment of Gram-positive infections in critically ill patients. BMC Infect Dis 2014;28:14-92.

35. von Eiff C, Becker K, Machka K, et al. Nasal carriage as a source of Staphylococcus aureus bacteremia. Study Group. N Engl J Med 2001;344:11-6. 